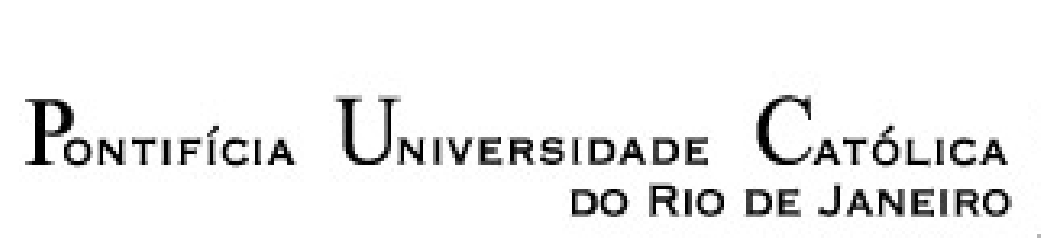

Mário Sérgio Sant'Anna Gonçalves

\title{
Aspectos da síntese por redução preferencial e caracterização do nanocompósito Co-ZnO
}

\section{Dissertação de Mestrado}

Dissertação apresentada como requisito parcial para obtenção do grau de Mestre em Ciências da Engenharia Metalúrgica pelo Programa de PósGraduação em Engenharia Metalúrgica do Departamento de Ciência dos Materiais e Metalurgia da PUC-Rio.

Orientador: Eduardo de Albuquerque Brocchi

Rio de Janeiro, março de 2005 


\section{Pontifícia Universidade Católica DO RIO DE JANEIRO}

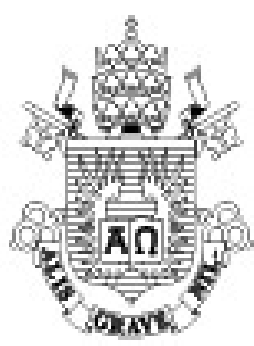

Mário Sérgio Sant'Anna Gonçalves

\section{Aspectos da síntese por redução preferencial e caracterização do nanocompósito Co-ZnO}

Dissertação apresentada como requisito parcial para obtenção do grau de Mestre em Ciências da Engenharia Metalúrgica pelo Programa de Pós-Graduação em Engenharia Metalúrgica do Departamento de Ciência dos Materiais e Metalurgia da PUC-Rio. Aprovada pela Comissão Examinadora abaixo assinada.

Prof. Eduardo de Albuquerque Brocchi Orientador Departamento de Ciências dos materiais e Metalurgia - PUC-Rio

Prof. Francisco Rolando Valenzuela Diaz USP

Prof. Francisco José Moura Departamento de Ciências dos materiais e Metalurgia - PUC-Rio

Prof. José Eugenio Leal Coordenador Setorial do Centro Técnico Científico - PUC-Rio

Rio de Janeiro, 28 de março de 2005 
Todos os direitos reservados. É proibida a reprodução total ou parcial do trabalho sem autorização da universidade, do autor e do orientador.

\section{Mário Sérgio Sant'Anna Gonçalves}

Graduou-se em Química Industrial pela FRPNL em 1993. Atuou em algumas empresas do setor químico. Graduourse pela segunda vez em Licenciatura Plena em Química pela UERJ em 2004. Publicou trabalho no XX ENTMME em 2004.

Ficha Catalográfica

Gonçalves, Mário Sérgio Sant’Anna

Aspectos da síntese por redução preferencial e caracterização do nanocompósito Co-ZnO / Mário Sérgio Sant'Anna Gonçalves ; orientador: Eduardo de Albuquerque Brocchi. - Rio de Janeiro : PUC-Rio, Departamento de Ciência dos Materiais e Metalurgia, 2005.

$81 \mathrm{f} . ; 30 \mathrm{~cm}$

Dissertação (mestrado) - Pontifícia Universidade Católica do Rio de Janeiro, Departamento de Ciência dos Materiais e Metalurgia.

Inclui referências bibliográficas

1. Ciência dos Materiais e Metalurgia - Teses. 2. Redução com hidrogênio. 3. Co-ZnO. 4. Caracterização. 5. Nanocompósito. I. Brocchi, Eduardo de Albuquerque. II. Pontifícia Universidade Católica do Rio de Janeiro. Departamento de Ciência dos Materiais e Metalurgia. III. Título. 
À minha esposa Leda e ao meu filho Thiago pelo apoio e incentivo. 


\section{Agradecimentos}

Em primeiro lugar a Deus, por mais uma vez ter consentido chegar até aqui. Sinceramente não saberia explicar como consegui.

Ao meu orientador Professor Eduardo de Albuquerque Brocchi pelo estímulo e parceria para a realização deste trabalho.

Ao CNPq e a PUC-Rio, pelos valiosos e fundamentais auxílios concedidos, sem os quais este trabalho não se concretizaria. Aos meus pais pela educação e carinho de todas as horas.

Ao meu irmão Mário Henrique e esposa, a tia Nini, aos amigos Renato e família Santiago. Aos professores que participaram da comissão examinadora. Mas diferentemente de outras ocasiões, tentarei fazer justiça a outras pessoas que passaram por este caminho, e que de uma forma ou outra, igualmente, contribuíram neste desafio.

Para tanto enumero:

Prof. Francisco Moura - DCMM.

Prof. Fathi Darwish - DCMM.

Prof. Roberto José de Carvalho - DCMM.

Prof. Roberto Avillez - DCMM.

Prof. Hélio Kohler - DCMM.

Prof. Guillermo Solórzano - DCMM.

Prof. Sidnei Paciornik - DCMM.

Prof. Maurício Torem - DCMM.

Prof. Raul Almeida - DCMM.

Prof.a. Maria Isabel Pais - DQ.

A todos os colegas com os quais convivi na PUC-Rio, a toda comunidade que presta serviços direta ou indiretamente, mantendo o bom funcionamento desta casa. Dentre os quais destaco:

Diógenes dos Santos Medeiros

Paulo Fernando Costa

Ronaldo Pedro da Silva

Vítor Hugo da Cunha

Carlos Augusto Queiroz

Amarildo Abrantes

Luzinete Patrício Araújo

Maurício Monteiro
BS/CTC.

Fotógrafo/DCMM.

Técnico/DCMM.

Técnico/DCMM.

Engenheiro/DCMM.

Técnico/DCMM-

Secretária/DCMM.

Pesquisador/DCMM. 


\section{Resumo}

Gonçalves, Mário Sérgio Sant'Anna . Aspectos da síntese por redução preferencial e caracterização do nanocompósito Co-ZnO. Rio de Janeiro, 2005. 81p. Dissertação de Mestrado - Departamento de Ciência dos Materiais e Metalurgia, Pontifícia Universidade Católica do Rio de Janeiro.

O trabalho aborda a elaboração do compósito $\mathrm{Co}-\mathrm{ZnO}$ por redução preferencial com hidrogênio. Inicialmente foi desenvolvida uma avaliação termodinâmica das principais reações para o processo tais como, a pirólise dos nitratos de cobalto e de zinco, responsável pela obtenção dos respectivos óxidos, e a redução destes pelo hidrogênio. $\mathrm{O}$ estudo experimental foi realizado de forma a se avaliar o efeito de variáveis importantes (fluxo, temperatura e pressão parcial do gás reagente) sobre a cinética das reações de redução. Observou-se a viabilidade de se estabelecer condições onde ocorresse a redução preferencial do óxido de cobalto em relação ao óxido de zinco. Como exemplo pode-se mencionar que na faixa de temperatura de realização dos experimentos de redução do óxido de cobalto $(590 \mathrm{~K}-790 \mathrm{~K})$, foram obtidas conversões entre 20 e $80 \%$, dentro de um intervalo de tempo compreendido entre 1 e 15 minutos e com fluxo de hidrogênio da ordem de $0,18 \mathrm{~L} / \mathrm{min}$. Por sua vez, o compósito pode ser obtido em ensaio realizado na temperatura de $690 \mathrm{~K}$ pelo tempo de 20 minutos mantido o mesmo fluxo. As caracterizações dos materiais obtidos foram realizados por difração de raios-X, microscopia eletrônica de varredura/EDS e microscopia eletrônica de transmissão, sendo possível identificar óxidos relativamente puros e o compósito $\mathrm{Co}-\mathrm{ZnO}$ bastante homogêneo e constituído por partículas na escala nanométrica, características estas, desejáveis para materiais desta natureza.

\section{Palavras-chave}

Redução com hidrogênio; Co-ZnO; caracterização; nanocompósito. 


\section{Abstract}

Gonçalves, Mário Sérgio Sant'Anna. Aspects of synthesis by preferential reduction and characterization of Co-ZnO nanocomposite. Rio de Janeiro, 2005. 81p. Dissertation - Departamento de Ciência dos Materiais e Metalurgia, Pontifícia Universidade Católica do Rio de Janeiro.

This work covers the $\mathrm{Co}-\mathrm{ZnO}$ nano-composite synthesis by hydrogen preferential reduction of these metals oxides which, in turn, were obtained by simultaneous dissociation of their nitrates. Initially it has been developed a thermodynamic evaluation of the main process reactions such as the zinc and cobalt nitrates pyrolises and the respective oxides reduction by hydrogen. The experimental work was carried out in order to study the effect of the most important variables on the reduction reaction kinetics of the cobalt oxide as well as to investigate some experimental conditions for the formation of the $\mathrm{Co}-\mathrm{ZnO}$ nanocomposite. It can be said that cobalt oxide is completely and preferentially reduced in 20 minutes at $690 \mathrm{~K}$, then allowing the composite formation at this temperature. The materials involved in the process were characterized by X-R diffraction (oxides and co-obtained oxides) and electronic microscopy (SEM/EDS, TEM), being possible to identify a Co-ZnO homogeneous and containing particles in the nano range scale.

\section{Key words}

Reduction with hydrogen; $\mathrm{Co}-\mathrm{ZnO}$; nanocomposite; characterization. 


\section{Sumário}

1 Introdução

2 Considerações teóricas

2.1. Aspectos termodinâmicos

2.2. Aspectos cinéticos

3 Metodologia experimental

3.1. Pirólise dos nitratos de cobalto e de zinco.

3.2. Redução dos óxidos

3.3. Caracterizações

4 Resultados e discussão

4.1. Pirólise dos nitratos de cobalto e de zinco.

4.2. Estudo cinético das reações de redução com hidrogênio dos óxidos de cobalto e de zinco.

4.2.1. Redução do óxido de cobalto pelo hidrogênio.

4.2.1.1. Efeito do fluxo do gás redutor sobre a redução do óxido de cobalto.

4.2.1.2. Efeito da temperatura sobre a cinética da redução do óxido de cobalto.

4.2.1.3. Efeito da pressão parcial de $\mathrm{H}_{2}$ sobre a conversão do óxido de cobalto.

4.2.2. Redução do óxido de zinco pelo hidrogênio

4.3. Elaboração do compósito Co-ZnO.

4.3.1. Pirólise da mistura dos nitratos de cobalto e de zinco.

4.3.2. Redução dos óxidos co-formados. 
6 Referências bibliográficas

Anexo I - Varistores

68

Anexo II - Gráficos e tabelas de calibração dos rotâmetros

72

Anexo III - Tabelas de valores das variáveis termodinâmicas das dissociações dos nitratos

75

Anexo IV - Tabelas de dados experimentais do estudo cinético das reduções do óxido de cobalto

76

Anexo V - Gráfico da análise térmica diferencial

79

Anexo VI - Lista de materiais

80 


\section{Lista de figuras}

Figura 1 Escala dos materiais naturais e os construídos pelo homem. 16

Figura $2.1 \log \mathrm{K}$ (constante de equilíbrio) versus temperatura (K). 21

Figura 2.2 $\Delta \mathrm{G}^{0}$ (variação de energia livre padrão da pirólise dos nitratos) versus $\begin{array}{ll}\text { temperatura }(\mathrm{K}) . & 22\end{array}$

Figura 2.3 $\Delta \mathrm{G}^{0}$ (variação da energia livre padrão de redução dos óxidos) versus temperatura $(\mathrm{K}) \quad 23$

Figura 2.4 Variação da pressão parcial de $\mathrm{H}_{2}$ versus temperatura (K). 24

Figura 2.5 Pressão parcial de $\mathrm{H}_{2}$ versus temperatura (K). 24

Figura 2.6. Composição das espécies no equilíbrio. 25

Figura 2.7 Variação das energias livres padrão dos redutores versus temperatura

(K). 26

$\begin{array}{ll}\text { Figura 3. } 1 \text { Chapa aquecedora } & 30\end{array}$

$\begin{array}{ll}\text { Figura 3.2Reator. } & 31\end{array}$

Figura 4.1 Difratograma do $\mathrm{Co}_{3} \mathrm{O}_{4}$. 34

Figura 4.2 Difratograma do ZnO. 35

Figura 4.3 - Imagem em alta resolução evidenciando os planos atômicos de $\mathrm{ZnO}$

Figura 4.4 Efeito da temperatura sobre a conversão do $\mathrm{Co}_{3} \mathrm{O}_{4}$. 38

Figura $4.5 \ln \mathrm{k}_{\mathrm{i}}$ versus 1/T (K) energia de ativação aparente da redução do $\mathrm{Co}_{3} \mathrm{O}_{4}$.

Figura 4.6 Efeito da temperatura sobre a conversão do $\mathrm{Co}_{3} \mathrm{O}_{4}$ submetido ao modelo topoquímico.

Figura 4.7 (ln ki versus 1/T) energia de ativação aparente da redução do $\mathrm{Co}_{3} \mathrm{O}_{4}$ submetido ao modelo topoquímico. $\quad 42$

Figura 4.8 Efeito da pressão parcial de hidrogênio sobre a redução do $\mathrm{CO}_{3} \mathrm{O}_{4} . \quad 44$

Figura 4.9 (ln r versus $\ln \mathrm{P})$. 45

Figura 4.10 Efeito da pressão parcial sobre a redução do Co3O4 submetida ao modelo topoquímico. 46

Figura 4.11 (ln r versus ln P) ordem de reação. $\quad 47$

Figura 4.12 Pressão de vapor dos materiais versus temperatura (K). 48 
Figura 4.13 Difratograma dos óxidos co-formados.

Figura 4.14 Conversão do óxido de cobalto a cobalto metálico ao longo do tempo na formação do compósito Co- $\mathrm{ZnO}$. 51

Figura 4.15 Difratograma do compósito Co-ZnO. 53

Figura 4.16 Difratograma do compósito Co-ZnO. 54

Figura 4.17 Caracterização MEV/EDS. 56

Figura 4.18 Par campo claro(a) e campo escuro (b) de um aglomerado de partículas de $\mathrm{Co}-\mathrm{ZnO}$, com uma dispersão bastante homogênea de tamanho médio de aproximadamente $30 \mathrm{~nm}$ e sua correspondente figura de difração em área selecionada (c). $\quad 57$

Figura 4.19 Espectro EDS das partículas Co-ZnO da Figura 4.18. 58 Figura 4.20 aglomerado hete rogêneo de partículas de $\mathrm{ZnO}$ reveladas no MET. Nas figuras (a) e (b) em campo claro, sob duas condições distintas de difração; (c) corresponde a figura de difração em área selecionada deste aglomerado; (d), (e) e (f) imagens em campo escuro utilizando diferentes reflexões do padrão da figura (c), iluminando diferentes cristais individuais $\mathrm{e}$ evidenciando defeitos. 


\section{Lista de tabelas}

Tabela 2.1 Valores das variáveis termodinâmicas da dissociação do nitrato de $\begin{array}{ll}\text { zinco. } & 75\end{array}$

Tabela 2.2 Valores das variáveis termodinâmicas da dissociação do nitrato de $\begin{array}{ll}\text { cobalto. } & 75\end{array}$

Tabela 4.1Efeito do fluxo do gás redutor sobre a redução do Co3O4. 76

Tabela 4.2 Efeito da temperatura sobre a conversão ao longo do tempo. 76

Tabela 4.3 Evolução da constante cinética com a temperatura nas reações de redução do $\mathrm{Co}_{3} \mathrm{O}_{4}$.

Tabela 4.4 Calculo da energia de ativação submetido ao modelo topoquímico. 77

$\begin{array}{ll}\text { Tabela 4.5 Efeito da pressão parcial do gás redutor. } & 77\end{array}$

Tabela 4.6 Efeito da pressão parcial do gás redutor submetido ao modelo topoquímico. $\quad 78$

Tabela 4.7 Conversões percentuais do óxido de zinco em função da temperatura. 